\title{
Oportunidades de Aprendizaje y Competencias Matemáticas: un estudio de dos casos
}

\section{Opportunities to Learn and Mathematics Competences: two case studies}

\author{
José Carrillo* \\ Luís C. Contreras ${ }^{* *}$ \\ Diana Zakaryan $^{* * *}$
}

\begin{abstract}
Resumen
Este estudio trata de conocer la importancia que tienen las oportunidades de aprendizaje (OTL) que ofrece el profesor en su aula a la hora de facilitar la adquisición de las competencias matemáticas de sus estudiantes (CM). Se presenta la parte empírica de una investigación, que ha consistido en el estudio de dos casos particulares (en España y Armenia), y documentamos los resultados obtenidos a la luz de un modelo teórico OTL-CM, construido de forma emergente en el proceso de análisis de los datos. Los resultados de nuestra investigación han constatado que las escasas OTL que promueven el aprendizaje significativo de las matemáticas limitan tanto la adquisición de los conocimientos y destrezas matemáticas básicas, como la capacidad de aplicarlos en situaciones de la vida real.
\end{abstract}

Palabras-clave: Oportunidades de Aprendizaje. Competencias Matemáticas. Relaciones entre oportunidades y competencias.

\begin{abstract}
This study is aimed at understanding the importance of the opportunities to learn (OTL) offered by a teacher in his classroom in facilitating the acquisition of mathematical competence (CM) of their students. In this paper we present the empirical part of this research, which consisted in the study of two cases (in Spain and Armenia) and documents the results in light of the theoretical model OTL-CM, which emerged in the analytical process. The results show that the scarcity OTL that promote meaningful learning of mathematics limits the acquisition of mathematical knowledge and basic skills, as well as the capacity to apply them to daily life situations.
\end{abstract}

Keywords: Opportunities to Learn. Mathematics Competences. Relationship between Opportunities and Competences.

\footnotetext{
${ }^{*}$ Doctor en Filosofía y Ciencias de la Educación por la Universidad de Sevilla (US), Sevilla, España. Profesor Titular de Universidad de Didáctica de la Matemática, Universidad de Huelva (UHU), Huelva, España. Dirección postal: Facultad de Ciencias de La Educación, Campus El Carmen, Avenida del 3 de Marzo, s/n, 21071, Huelva, España. E-mail: carrillo@uhu.es.

${ }^{* *}$ Doctor en Psicopedagogía por la Universidad de Huelva (UHU), Huelva, España. Profesor Titular de Universidad de Didáctica de la Matemática, Universidad de Huelva (UHU), Huelva, España. Dirección postal: Facultad de Ciencias de La Educación, Campus El Carmen, Avenida del 3 de Marzo, s/n, 21071, Huelva, España.E-mail:1carlos@uhu.es.

* Doctora por la Universidad de Huelva (UHU), Huelva, España. Profesora Asociada de Didáctica de la Matemática, Pontificia Universidad Católica de Valparaíso (PUCV), Valparaíso, Chile. Dirección postal: Instituto de Matemáticas, Pontificia Universidad Católica de Valparaíso, Blanco Viel 596, Cerro Barón, Valparaíso, Chile. E-mail: diana.zakaryan@ucv.cl.
} 


\section{Introducción}

En este artículo, que es continuación de Carrillo, Contreras y Zakaryan (2013), presentamos los datos empíricos de una investigación (ZAKARYAN, 2011), que ha tratado de comprender cómo las oportunidades de aprendizaje (OTL) que ofrecen los profesores a los estudiantes se relacionan con sus competencias matemáticas (CM).

El concepto de Oportunidades de Aprendizaje ha desempeñado, durante varios años, diferentes papeles: como concepto investigativo (research concept), como indicador de la educación (education indicator) y como instrumento político (policy instrument) (MCDONNELL, 1995) y actualmente, según Hiebert y Grouws (2007) se considera como uno de los pocos indicadores que conectan la enseñanza y el aprendizaje, y pretende explicar las diferencias en el aprendizaje de matemáticas de los estudiantes a través de varios contextos. Esta acepción es utilizada en este artículo.

Dentro del estudio de OTL, aquí nos centramos en el papel del profesor a la hora de determinar dichas oportunidades (STEVENS; GRYMES, 1993), particularmente, en las actividades del profesor en el aula: el foco matemático (FM), las estrategias didácticas (ED) y el tipo de tareas (TT) (ANDREWS; CARRILLO; CLIMENT, 2005), que condicionan la naturaleza de la enseñanza que desarrolla. La segunda línea de investigación de este estudio tiene que ver con la noción de Competencias Matemáticas, en la cual nos situamos en el marco del proyecto PISA 2003 (OCDE, 2004).

A partir de estos dos marcos (su detallada descripción se puede consultar en Zakaryan (2011) y Carrillo, Contreras y Zakaryan (2013)) elaboramos un modelo OTL-CM que trata de describir, explicar y predecir los fenómenos de la enseñanza-aprendizaje, en términos de que ciertas oportunidades de aprendizaje que ofrecen los profesores en sus aulas pueden favorecer el desarrollo de ciertas competencias matemáticas. En la construcción del modelo consideramos una trayectoria hipotética de enseñanza (THE) de las matemáticas. A partir de las Trayectorias Hipotéticas de Aprendizaje de Simon (1995), usamos trayectoria hipotética de enseñanza (THE) para referirnos a la supuesta evolución en la enseñanza del profesor según cambian sus objetivos. Por otra parte, en nuestro modelo, los objetivos del profesor igualmente determinan sus actividades, sin embargo, no consideramos explícitamente sus hipótesis acerca del proceso de aprendizaje, sino las relacionamos con la potencialidad de desarrollar ciertas competencias matemáticas de los estudiantes.

Determinamos tres niveles de THE, que dependiendo del objetivo de aprendizaje del profesor, proporcionan diferentes oportunidades de aprendizaje asociadas a las categorías 
antes mencionadas y sus correspondientes subcategorías (en Zakaryan (2011) y Carrillo, Contreras y Zakaryan (2013), describimos y justificamos sus correspondencias). Relacionamos estos tres niveles de THE con el eje principal del modelo constituido por las siete competencias matemáticas (pensar y razonar, argumentar, comunicar, modelizar, plantear y resolver problemas, representar y utilizar el lenguaje simbólico, formal y técnico y las operaciones) o procesos cognitivos (y sus clasificaciones según los tres grupos de reproducción, conexión y reflexión) que activan los estudiantes al abordar problemas matemáticos (OCDE, 2003, 2004), presentados como un continuo: nivel bajo-nivel medionivel alto. Reflejamos estas relaciones mediante el modelo OTL-CM (Figura 1).

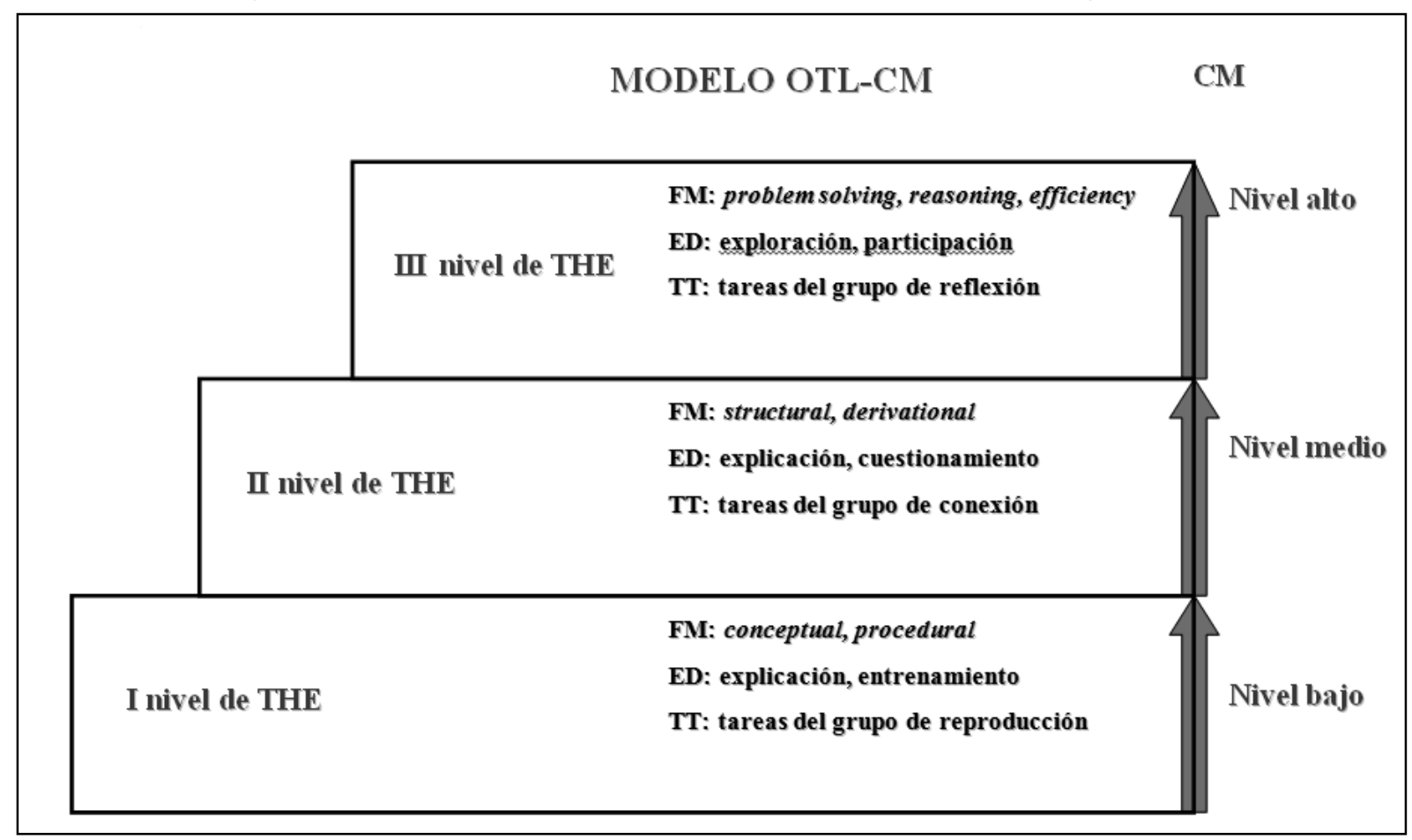

Figura 1 - Modelo OTL-CM (FM, foco matemático; ED, estrategias didácticas; TT, tipo de tarea) Fuente: desarrollado por los autores

A la hora de interpretar los resultados tenemos siempre presente los tres niveles en los objetivos de aprendizaje del profesor.

En las siguientes líneas exponemos el proceso de obtención de los datos empíricos, su análisis y los resultados.

\section{Metodología}

Resulta complejo tratar de comprender cómo las oportunidades de aprender (OTL) que ofrecen los profesores a los estudiantes se relacionan con sus competencias matemáticas (CM), pues supone una comprensión de los cómo y por qué de procesos de enseñanza y Bolema, Rio Claro (SP), v. 28, n. 48, p. 89-109, abr. 2014 
aprendizaje de las matemáticas. Precisamente por ello, lo abordamos a través de un estudio de casos (STAKE, 2007), combinando distintos métodos cualitativos y cuantitativos (BERICAT, 1998). Nuestro interés reside sobre todo en aprender de los casos estudiados, obteniendo mayor comprensión sobre la pregunta planteada a través de un estudio instrumental de casos (STAKE, 2007), donde el caso es un instrumento para adquirir conocimiento y comprensión sobre la temática determinada con el fin de generar o refinar teorías. Hemos decidido estudiar dos casos, que corresponden a dos contextos y tradiciones de enseñanza diferentes, con la finalidad de obtener información diversa y rica en significados. En cada caso nos centramos en el estudio de un aula ${ }^{1}$, una de ellas ubicada en un centro de Educación Secundaria (ESO) de Huelva (España) y la otra en una escuela de Ereván (Armenia); ambas elegidas según el interés de la investigación, a las que acuden estudiantes entre 15-16 años (la edad en la que la mayoría de estudiantes termina la enseñanza obligatoria).

Nuestro diseño, en general, ha seguido un plan establecido en cuanto al acceso al escenario, la permanencia en el mismo y la retirada del escenario; sin embargo, ha permanecido constantemente evolucionando y reelaborándose a partir del estudio de los datos obtenidos a la luz de su reducción, categorización y elaboración del modelo teórico.

De este modo, en los siguientes apartados contextualizamos los dos casos, tratando Caso 1 cuando nos referimos al aula de $4^{\circ}$ ESO de Huelva y Caso 2 al de IX ${ }^{\circ}$ grado de una de las escuelas de Ereván, y presentamos diferentes técnicas de recogida y de análisis de información.

\subsection{Descripción de casos}

Caso 1: El profesor, a quien en adelante, llamaremos $\mathrm{Pablo}^{2}$, en aquel momento tenía 29 años de experiencia como docente en el mismo centro e impartía clases de matemáticas en $4^{\mathrm{o}}$ de ESO (opción B). Su formación inicial es ingeniero-técnico de minas. Sus estudiantes (curso 2006/2007), entre 15-16 años, fueron elegidos por nuestro interés en conocer las competencias de los estudiantes de dicha edad. Los 15 estudiantes de esta aula que participaron en el estudio proceden de familias del nivel social económico y cultural medio bajo. El rendimiento curricular (promedio del aula) en matemáticas de estos estudiantes

\footnotetext{
${ }^{1}$ Dado que los casos son las aulas, elegidas por facilidad de acceso, disponibilidad de sus integrantes y con estudiantes de 15 años, los profesores estudiados son, justamente, los asignados a los cursos analizados.

${ }^{2}$ Todos los nombres son pseudónimos, para preservar sus identidades reales. 
durante los tres primeros años de Secundaria se aproxima a la puntuación de Notable, decreciendo en el cuarto año hasta la de Suficiente.

Caso 2: La profesora, en adelante Mery, ha terminado dos carreras: Licenciatura en Matemáticas Aplicadas y se tituló como Profesora en Físicas y Matemáticas por la Universidad Pedagógica. En aquel momento tenía 12 años de práctica en el centro y mantenía el mismo grupo de estudiantes a partir de $\mathrm{IV}^{\mathrm{o}}$ grado. Los estudiantes del IX ${ }^{\mathrm{o}}$ grado de curso académico 2008/2009, son jóvenes entre 15-16 años, como en el Caso 1, han sido elegidos intencionadamente. En el estudio participaron 25 estudiantes que procedían de familias del nivel social económico y cultural medio alto. El rendimiento curricular (promedio del aula) en matemáticas de estos estudiantes durante los últimos cuatro años de sus estudios oscila alrededor de la puntuación de Notable.

\subsection{Proceso e instrumentos de obtención de la información}

Con el fin de construir e interpretar la realidad, objeto de investigación, recurrimos a diferentes técnicas de obtención de los datos dependiendo del informante. Consideramos la observación de aula (COHEN; MANION, 2002) como la fuente principal de la información $\mathrm{y}$, en ambos casos, realizamos las observaciones de aula a través del método de observación no participante, que ha supuesto la presencia de la investigadora en el aula, sin intervención alguna. Durante las grabaciones en vídeo de las mismas, nos interesaba captar tanto las intervenciones del profesor como las de los estudiantes, por lo que la cámara ha sido colocada en la parte trasera del aula.

La técnica de recogida de la información, acerca de las competencias matemáticas de los estudiantes, ha sido una prueba de matemáticas. Dicha prueba de papel y lápiz, con la duración de 1 hora y 40 minutos, ha consistido en 39 problemas liberados de los de PISA 2003 (INECSE, 2005). Decidimos realizar la prueba al final del año académico correspondiente en cada caso, con el propósito de que los estudiantes tuvieran la oportunidad de cursar la mayor parte posible de la programación establecida para el curso.

\subsection{Proceso e instrumentos de análisis de la información}

Tras la transcripción y organización de la información obtenida, realizamos su análisis, en cada caso, tanto de las OTL puestas en juego como de las CM mostradas, como se describe a continuación. 


\subsubsection{El instrumento de análisis de las OTL}

En este apartado damos sentido a la información obtenida de las sesiones videograbadas en ambos casos, tratándola desde la perspectiva de las OTL que proporcionan Pablo y Mery, en sus correspondientes aulas, cuando enseñan matemáticas.

Con este fin, dividimos las sesiones en episodios que representan las oportunidades dadas a los estudiantes. Entendemos por episodio un fragmento de la sesión en el que la intención relativa a los objetivos de aprendizaje del profesor ha sido constante (p.ej. trabajo en la resolución de problemas, introducción de los conceptos, ejercitación de las fórmulas). Los objetivos del profesor los identificamos a partir de las actividades que realiza en el aula. De este modo, analizamos cada episodio según las categorías mencionadas: foco matemático, estrategias didácticas, y tipo de tareas propuestas por el profesor.

Tal como hemos mencionado, codificamos las sesiones grabadas en ambos casos mediante las categorías expuestas y realizamos un análisis profundo de la información con el fin de generar una comprensión del problema planteado.

Por otra parte, para organizar los datos en unidades de información, recurrimos al esquema de representación del modelo propuesto por Schoenfeld (1998). De esa representación gráfica, nos interesa utilizar el esquema mismo para organizar los episodios $\mathrm{j}$ de una sesión concreta $\mathrm{i},[\mathrm{i}, \mathrm{j}] \mathrm{y}$ describir los eventos iniciales de un episodio y los que funcionan como causa del fin del mismo, el objetivo del profesor. Por lo demás, seguimos nuestra meta de identificar los indicadores de las OTL, anteriormente establecidos. La Ilustración 1 representa el esquema que seguiremos para el análisis de las sesiones grabadas.

[i.j] Designación del episodio (línea de inicio - línea de fin)

Evento desencadenante: Evento que funciona como desencadenante de las secuencias de actividades.

OPORTUNIDADES DE APRENDIZAJE:

Identificación de las oportunidades de aprendizaje subyacentes en esta secuencia de actividades.

Foco matemático (conceptual, procedural, structural, derivational, efficiency, problem solving, reasoning)

Estrategias didácticas (explicación, participación, exploración, entrenamiento, cuestionamiento)

Tipo de tareas (procesos cognitivos, situaciones y contextos)

Evento de término:

Evento que funciona como causa de término de la secuencia de actividades.

Ilustración 1 - Representación del modelo de análisis de las sesiones

Fuente: desarrollado por los autores 


\subsubsection{El instrumento de análisis de las $\mathrm{CM}$}

La fuente principal para análisis de las competencias matemáticas son los resultados de la prueba aplicada.

En primer lugar, realizamos el análisis de protocolos de resolución de cada ítem desde el enfoque de análisis de contenido (BARDIN, 1996), a la luz de las CM (los procesos cognitivos) que han sido capaz de activar y/o las que han resultado imposible poner en marcha durante sus actuaciones. Por último, corregimos los trabajos de los estudiantes según los criterios y códigos establecidos para los ítems liberados de la prueba PISA 2003, los analizamos cuantitativamente según el modelo de $\operatorname{Rasch}^{3}$ y los categorizamos por los 6 niveles de dominio (OCDE, 2003). Esta categorización de las respuestas nos permite conocer los niveles en el dominio de los estudiantes de acuerdo con la escala establecida en la prueba PISA 2003 y, además, nos ayuda a ubicarlos en el continuo de niveles bajo-medio-alto a la hora de interpretar los resultados a la luz de las relaciones determinadas en el modelo OTLCM.

\section{Análisis de información}

\subsection{Análisis de las OTL}

En el Caso 1, observamos en total 7 sesiones relacionadas a los tópicos de Elementos de geometría. En la Ilustración 2 presentamos un ejemplo del análisis del segundo episodio de la segunda sesión [2.2].

\footnotetext{
${ }^{3}$ Para la elaboración de los datos usamos el programa Winsteps (LINACRE, 2006) y escalamos nuestro test en la métrica de la prueba PISA según los procedimientos básicos para establecer el escalamiento común (PRIETO; DÍAS, 2003). 


\section{[2.2]Trabajo en tareas [391-435]}

Evento desencadenante: Pablo escribe en la pizarra fórmulas y propone realizar actividades para aplicarlas.

OPORTUNIDADES DE APRENDIZAJE:

Foco matemático

Procedural - Después del intento fracasado de invitar a los estudiantes realizar la actividad, Pablo muestra en la pizarra cómo realizar los procedimientos para llevarla a cabo [408-424].

Estrategias didácticas

Entrenamiento - Pablo explícitamente ofrece consejos e indicaciones para realizar la actividad [408-424].

Tipo de tarea

N5 En este triángulo rectángulo (se da el dibujo con los catetos dados 7 y 10, la altura h y las proyecciones de los catetos sobre hipotenusa $\mathrm{m}$ y n), calcula las longitudes h, $\mathrm{m}$ y n (p.159).

Procesos cognitivos: Reproducción

Situación: Científica

Contexto: Hipotético

Evento de término: Fin de la sesión.

\section{Ilustración 2 - Análisis del segundo episodio de la segunda sesión de Pablo}

Fuente: desarrollado por los autores

En las sesiones de Pablo se enfatizan procedimientos y técnicas de cálculo y el desarrollo conceptual de los estudiantes. Sin embargo, este último tiene más bien carácter formal, ya que los conceptos abordados durante las sesiones se presentan como hechos acabados y unidades de información aisladas.

De este modo, por ejemplo, a pesar de que los conceptos relacionados con la semejanza y el teorema de Thales están presentes en numerosas situaciones del mundo real (fotografías, proyecciones de imágenes o máquinas fotocopiadoras, o proyección de sombras), excepto el ejemplo de escala ilustrado en el libro de texto, Pablo no había puesto ningún otro, ni había invitado a los estudiantes a buscar ejemplos relacionados con la vida cotidiana, para encontrar en la realidad campos de aplicación oportunos para mejorar la comprensión de estos conceptos y establecer conexiones con su experiencia. De la misma manera formal se trata la proporcionalidad de áreas y volúmenes de figuras geométricas: se constata como hecho que la razón de semejanza para estas medidas es, respectivamente, el cuadrado y el cubo de la razón de semejanza.

P: [...] ¿Qué ocurre con las áreas? No sé si hemos dicho cuando estuvimos explicando el tema, pues las áreas son también proporcionales, solo la razón es igual a cuadrado de la razón de semejanza, ¿vale? [908-913].

Durante el análisis, observamos los fundamentos teóricos se ven con rapidez, dado que Pablo tiende a considerar la teoría como herramienta o recurso para la práctica, y la importancia que atribuye al foco procedural se refleja durante sus sesiones, sobre todo, cuando los estudiantes no son capaces de ejecutar alguna actividad. Pablo repite las técnicas y 
los procedimientos, y en varias ocasiones le sorprende que los estudiantes no sean capaces de aplicar fórmulas después de que haya explicado el procedimiento una y otra vez.

P: La regla siempre sigue la misma, yo lo que no entiendo es que una vez sale bien y otra vez sale mal, no lo entiendo, o sale todo mal o todo bien porque la aplicación es siempre igual [725-727].

Pablo ha usado con mayor frecuencia las estrategias didácticas explicación y entrenamiento. Un rasgo muy propio observado en todas las sesiones analizadas es su modo de introducir un nuevo concepto, teorema, criterios etc., que consistía en que alguno de sus estudiantes leía sus enunciados en el libro de texto y luego le seguía la explicación de Pablo.

P: Vamos a ver que nos dice teorema de Thales [...] ¿Adrián quieres leer? (Lee del libro). Bien, vamos a ver, aqui lo que nos dice es que tenemos dos rectas cortadas a su vez por tres rectas paralelas, entonces estas tres rectas determinan segmentos, entonces dice, ... Esto lo que está diciendo [154-164].

La explicación de Pablo, en algunos casos, se presentaba como reproducción literal del libro de texto (ejemplo anterior) y, en otros, como transmisión verbal con exposiciones durante la cual hacía preguntas como si fueran dirigidas a los estudiantes y contestaba él mismo, aclarándolas, de tipo monólogo.

La intervención del profesor durante el proceso de la resolución ha sido permanente y ha consistido en dar consejos e indicaciones de corregir; muchas veces, la tarea quedaba resuelta por él. Normalmente, Pablo usaba una secuencia de preguntas que requerían de los estudiantes identificación y reproducción, tales como: ¿Qué es?, ¿cuál aplico?, ¿cómo sería?, ¿cuál será?, ¿cuánto mide?.

De acuerdo con el análisis de las sesiones grabadas, el Gráfico 1 visualiza el perfil de Pablo según las OTL consideradas en nuestro estudio.

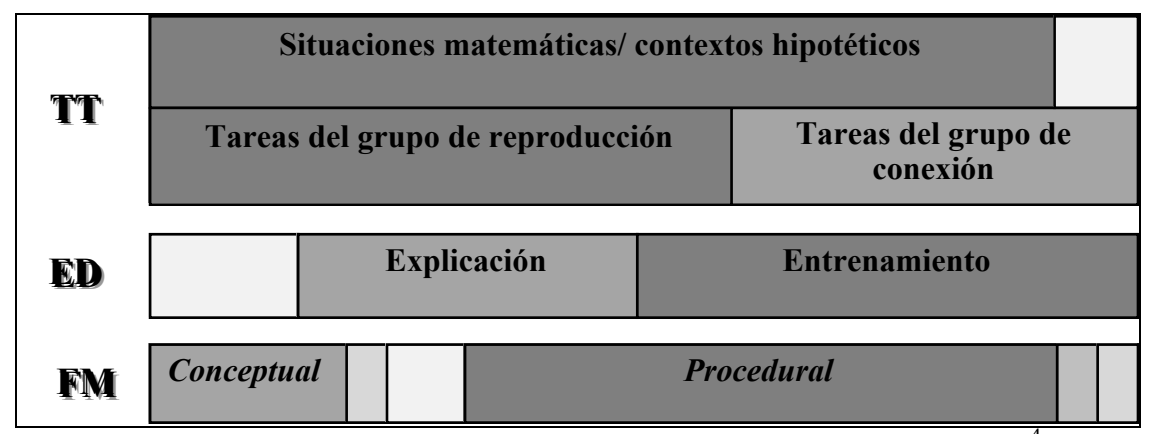

Gráfico 1 - Perfil del profesor Pablo según las OTL consideradas ${ }^{4}$ Fuente: desarrollado por los autores

\footnotetext{
${ }^{4}$ Destacamos en tonos oscuros las subcategorías mayormente observadas, los otros tonos que aparecen en el gráfico se relacionan con el resto de las subcategorías determinadas para cada una de las categorías (FM, ED, TT). Por otro lado, la barra inferior de TT se relaciona con los procesos cognitivos, y la superior con las situaciones y contextos.
} 
De este modo, Pablo ha enfatizado los procedimientos y conceptos, por medio de entrenamiento y explicación; ha manejado, mayormente, tareas del grupo de reproducción, pero también del grupo de conexión, planteadas en situaciones matemáticas y contextos hipotéticos. Además, Pablo ha utilizado trabajo en grupo-clase o individual y ha recurrido al libro de texto como al material didáctico único.

En el Caso 2, observamos 6 sesiones; una unidad didáctica del tema Concepto de logaritmo de Álgebra y Elementos de Análisis Matemático, y una de Vectores en el espacio de Geometría.

El análisis de las clases grabadas muestra que las sesiones de Mery se caracterizan por un cierto equilibrio de los focos matemáticos. Mery presenta los hechos y conceptos como parte de un todo, subrayando las conexiones entre diferentes nociones y entes matemáticos e infiriendo sus propiedades. No obstante, los lazos y relaciones se establecen, exclusivamente, dentro del área de la Matemática; por ejemplo, en ningún momento se menciona dónde y cómo se puede aplicar la función logarítmica (ni cualquier otra función que habían tratado) en las situaciones de la vida real o qué procesos permite describir. Análogamente, al introducir el concepto de vectores en el espacio, Mery pone ejemplos de Física de los vectores en el plano y en el espacio para enfatizar los lazos entre los conceptos matemáticos y los de Física.

P: [...] Por ejemplo, de los vectores en el plano, vosotros habiais pasado valores vectoriales de fisica tales como la velocidad, fuerza. ¿Y del espacio qué vectores habéis pasado? Por ejemplo, particulas cargadas de tensión (electricidad), E ¿no? [...].

De este modo, los hechos y conceptos se manejan y tienen sentido, exclusivamente, en el marco de la asignatura o en el de las materias afines.

Durante sus clases, Mery presta especial atención al hecho de que los estudiantes organicen bien los datos del problema y procedan en su resolución paso por paso; que al dibujar los cuerpos geométricos traten de visualizar todos sus elementos y utilicen diferentes estrategias (efficiency), entre ellas las del cálculo mental, para facilitar rapidez de las operaciones.

El fragmento que sigue ejemplifica cómo Mery promueve el desarrollo de la argumentación y razonamiento en sus estudiantes:

$P$ : ¿Cuál sería el vector colineal al vector nulo?

Es: Cualquiera.

P: ¿Por qué? 
E: Porque en el plano por el punto que no pertenece a la recta dada siempre se puede pasar una recta paralela a la dada [481-486].

A partir del análisis realizado, en el Gráfico 2 presentamos el perfil de Mery según las OTL consideradas.

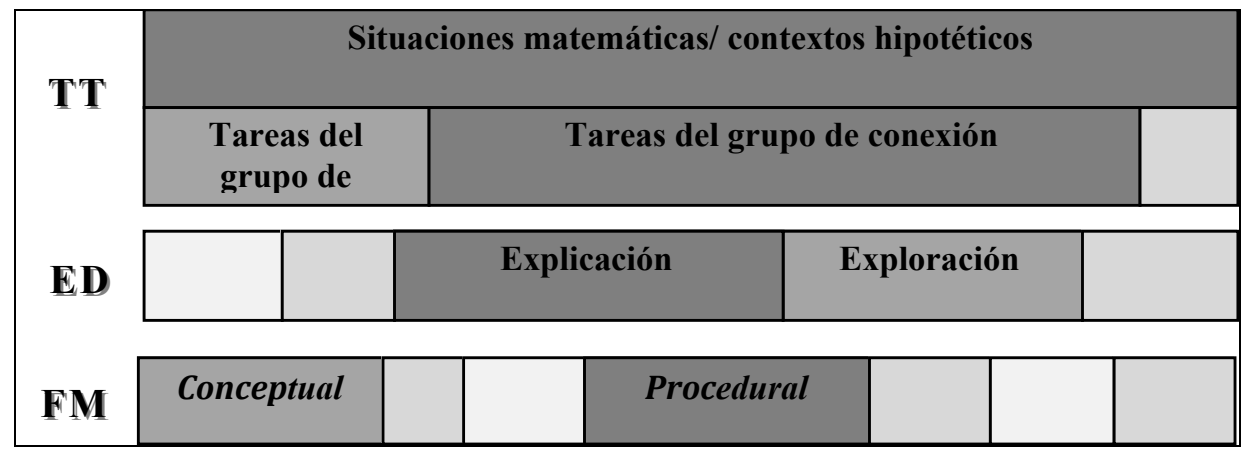

Gráfico 2 - Perfil de la profesora Mery según las OTL consideradas Fuente: desarrollado por los autores

De este modo, durante sus clases, Mery recurre a diversidad de focos matemáticos, con mayor énfasis relativo en conceptos y procedimientos, pero también en los demás focos. Usa, prioritariamente, las estrategias didácticas explicación, exploración, entrenamiento, cuestionamiento; propone trabajar, mayormente, en tareas del grupo de conexión, planteadas, exclusivamente, en situaciones científicas y contextos hipotéticos. Asimismo, trabaja con el grupo-clase o individual y usa el libro de texto como material didáctico principal.

\subsection{Análisis de las CM}

El análisis de los protocolos de los estudiantes ha revelado sus dificultades y carencias a la hora de resolver problemas realistas, dificultades que en principio, reflejan, o bien un vacío importante en los conocimientos matemáticos básicos, o bien la incapacidad de aplicarlos en situaciones de la vida real. Como consecuencia, los procedimientos y operaciones respectivas se han llevado a cabo erróneamente o no han llegado a realizarse. Asimismo, han mostrado dificultades en el uso del lenguaje simbólico, esto es, en identificar los datos con las variables de una fórmula dada o traducir el lenguaje simbólico al lenguaje coloquial. Del mismo modo, han sabido hacer inferencias directas y explicar asuntos matemáticos sencillos, sin llegar a formular supuestos o explicar asuntos matemáticos complejos que implican relaciones.

Por otra parte, en el Caso 1 notamos un acercamiento mecánico a la resolución de problemas por la mayoría de los estudiantes, hasta los más competentes, en el sentido evaluado, parecen caer en la tentación de realizar operaciones mecánicamente, sin 
interpretación matemática adecuada. Por ejemplo, se ha observado un error común en despejar la incógnita $P$ de la fórmula dada $\left(\frac{70}{P}=140\right)$. Aparte de la carencia en la técnica de cálculo, este hecho subraya el acercamiento mecánico, sin entrar en detalles y sin una interpretación adecuada de los resultados, ya que en caso contrario, al obtener una longitud del paso de dos metros (según sus cálculos), los estudiantes podrían cuestionar el resultado y volver a repensar la solución.

En el Caso 2 observamos la clara preferencia que muestran los estudiantes a la hora de abordar los ítems de la prueba: han buscado los ítems en los que haya sido explicita la operación o procedimiento matemático requerido (p.ej. aplicar porcentaje directo), evitando abordar los ítems donde había que interpretar un diagrama y explicar o argumentar su respuesta, lo que refleja la seguridad y familiaridad en unos e inseguridad en otros tipos de exigencias del enunciado. Asimismo, los estudiantes han tenido dificultad en entender el enunciado de los ítems, lo expresaban sus caras y los espacios que han dejado en blanco.

Las observaciones realizadas en el Caso 1 ponen de manifiesto que incluso la actuación competente en la prueba de algunos estudiantes tiene poco que ver con sus actuaciones durante las clases de matemáticas asistidas y grabadas en video. Estos estudiantes, igual que los demás, mantenían una actitud pasiva, no se sentían seguros en sus respuestas o al ejecutar operaciones de las tareas propuestas, mostrando inseguridad y lentitud bastante notable al manejar números o expresiones matemáticas.

Por otra parte, durante las observaciones que realizamos en el Caso 2, hemos visto a estudiantes más implicados, la mayoría supo abordar las tareas propuestas; se sentían más seguros a la hora de manejar los símbolos, operaciones y procedimientos matemáticos, de la misma forma que supieron razonar acerca de asuntos matemáticos, justificando su respuesta haciendo referencia a un teorema, axioma o propiedad. Estos estudiantes han tenido más éxito a la hora de resolver problemas puramente matemáticos incluso complejos, que al abordar los ítems de la prueba más sencillos. Por ejemplo, el ítem, en el que a partir de la representación gráfica de dos funciones que describen la estatura media de los chicos y las chicas de Holanda en 1998, han de señalar durante qué periodo de su vida son las chicas más altas que los chicos de su misma edad, la mayoría de los estudiantes no lo ha sabido abordar sin embargo, han sabido construir gráficos de funciones más sofisticadas, determinar su dominio, intervalos de monotonía, etc.

De este modo, el Gráfico 3 refleja el porcentaje de estudiantes por niveles de dominio para el Caso 1 y Caso 2. 


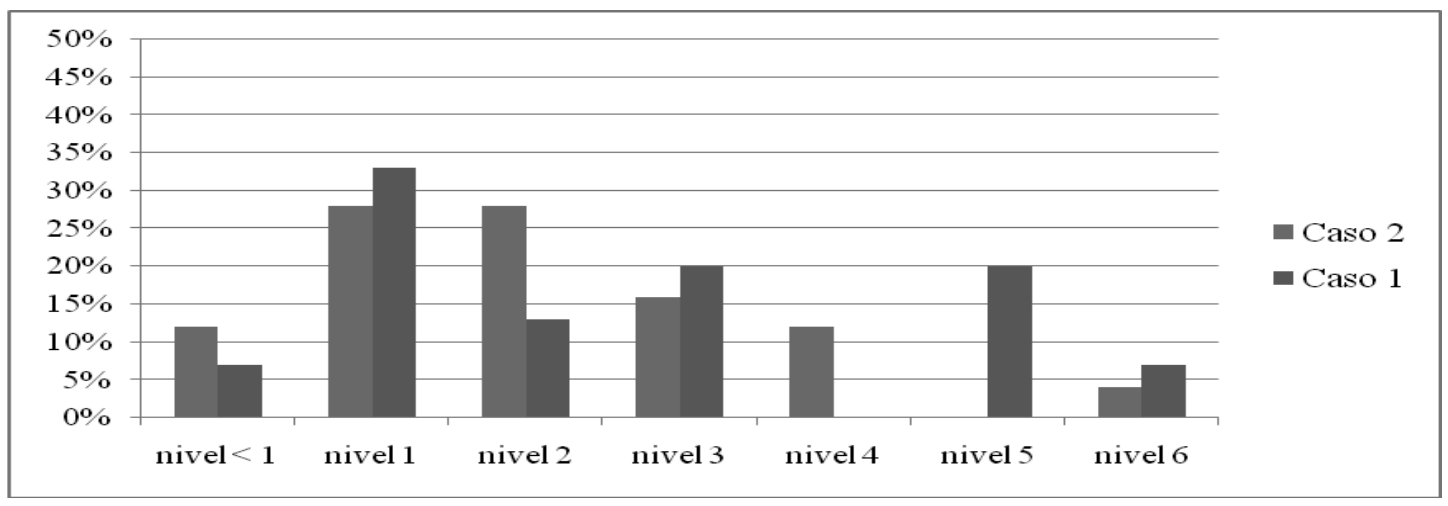

Gráfico 3 - Porcentaje de estudiantes según niveles de dominio (Caso 1, Caso 2)

Fuente: desarrollado por los autores

El 46\% (56\%) de estudiantes han mostrado nivel bajo de dominio (nivel 1 y 2), son capaces de resolver problemas elementales que requieren cálculos mecánicos, ejecución de procedimientos rutinarios, aplicación de destrezas técnicas y de algoritmos habituales. Finalmente, un $7 \%$ (12\%) de estudiantes tienen el nivel del rendimiento insuficiente y la falta de desarrollo de destrezas matemáticas (nivel $<1$ ).

\section{Resultados y discusión}

En este apartado exponemos los resultados sobre las relaciones entre las OTL y las CM y nuestro discurso al respecto para el Caso 1 y Caso 2.

\subsection{Resultados y discusión Caso 1}

Para el Caso 1, documentamos, por un lado, las oportunidades de aprendizaje enfocadas en técnicas y operaciones, centradas en el profesor y en la ejecución de tareas planteadas, mayormente, en situaciones matemáticas, mediante enfoques rutinarios, y, por otro lado, el bajo nivel de dominio de los estudiantes.

De este modo, de acuerdo con el modelo de OTL-CM elaborado, las oportunidades de aprendizaje proporcionadas a los estudiantes corresponden a las del primer nivel de la THE que, según las relaciones consideradas, no favorecen a la formación de las competencias matemáticas.

Las dificultades y carencias de los estudiantes, tanto a la hora de trabajar en el aula como al resolver problemas realistas, reflejan un vacío importante en los conocimientos matemáticos básicos y destrezas, resultado plausible de una enseñanza en la que los estudiantes han tenido la oportunidad de aprender formalmente los conceptos como unidades 
de información carentes de significado, sin una profundidad debida, ausente de conexiones entre diferentes entes matemáticos, o bien, sin una fijación adecuada de lo aprendido; de aplicar procedimientos y operaciones de una manera mecánica; de resolver ejercicios tipo o principalmente utilizando enfoques estándar, o sea, han tenido escasas oportunidades para el aprendizaje significativo de las matemáticas.

Por otra parte, como podemos ver en el análisis de las sesiones de Pablo, se trata de una enseñanza basada en la exposición magistral de los temas sucesivos del libro de texto a grupo-clase, prioritariamente, mediante las estrategias didáctica entrenamiento y explicación. El papel de Pablo en el aula es central: protagoniza todo el proceso casi en modo exclusivo, principalmente, explicando los tópicos, procedimientos y soluciones; su intervención se observa constantemente durante el trabajo en las tareas propuestas: muchas veces lee su enunciado, organiza los datos en la pizarra, representa el dibujo correspondiente, da pistas y consejos directos para su proceder, y, en varias ocasiones, termina resolviendo él. Este tipo de protagonismo limita las oportunidades de los estudiantes para aprender a traducir el enunciado de las tareas, a plantear y resolver problemas, a representar los objetos matemáticos, a expresar sus ideas.

A su vez, el papel del estudiante, en general, se reduce a escuchar, leer del libro de texto las definiciones, teoremas y criterios, y seguir las indicaciones del profesor. Como notamos en nuestras observaciones, una de las razones de la incapacidad de los estudiantes de aplicar los conceptos y teoremas impartidos, es su papel pasivo en la adquisición de los conocimientos. Es sabido que el papel pasivo del estudiante conlleva una comprensión pasiva, o sea, reconocimiento y reproducción de los hechos, frente a una comprensión activa cuando es capaz de evocar y utilizar lo aprendido (PIAGET, 1973; MIALARET, 1984).

Según el análisis del tipo de tareas propuestas por el profesor en sus sesiones, Pablo ha proporcionado la oportunidad de trabajar, mayormente, en tareas del grupo de reproducción, planteadas en situaciones científicas (matemáticas) y contextos hipotéticos. No ha dado la oportunidad de resolver problemas con demanda cognitiva de alto nivel, experimentar sus habilidades en varias situaciones y contextos. Esto explica que los procesos de pensamiento, que utilizan los estudiantes durante la resolución de este tipo de tareas, abarquen tan solo la elección de los datos, la aplicación de fórmulas y algoritmos estándar, el uso de una sola estrategia de solución, la identificación de representaciones sencillas y de una única manera, y la comunicación de los resultados de forma cerrada.

Tal como mencionamos en el modelo OTL-CM, si los estudiantes han tenido la oportunidad de realizar, principalmente, tareas de demanda cognitiva inferior (reproducción), 
es poco probable que adquieran procesos propios a la actividad matemática tales como observación, análisis y síntesis, generalización, abstracción, verificación; por tanto, es plausible que encuentren dificultad a la hora de abordar tareas matemáticas más complejas, distintas de las que resuelven en el aula, aquellas que no les resultan familiares y que requieren unos procedimientos diferentes de los usados.

Por otra parte, las tareas son planteadas, prioritariamente, en situaciones matemáticas, aunque a veces se presenten algunas actividades en situaciones de la vida con el pretexto de realizar alguna operación matemática. Siendo únicamente éstas las tareas planteadas en situaciones de la vida real en contextos hipotéticos, no se ha dado la oportunidad de desarrollar habilidades de matematización, precisamente las que necesitaban los estudiantes para abordar problemas realistas de la prueba.

Es cierto que las oportunidades de aprendizaje, observadas e inferidas en el Caso 1, no han favorecido el desarrollo de las competencias matemáticas, sino que éstas han resultado limitadas, reducidas a la reproducción, memorización y aplicación mecánica. A pesar de que, según la programación, los estudiantes conocían los contenidos matemáticos de la prueba, la adquisición superficial de los conocimientos y destrezas, la falta de experiencias en movilizar y aplicar sus conocimientos en una variedad de situaciones y contextos, mediante resolución de problemas de demanda cognitiva cada vez más alta, no ha favorecido a la actuación competente de los estudiantes.

\subsection{Resultados y discusión Caso 2}

A partir del análisis de la información para el Caso 2, observamos, por un lado, las oportunidades de aprendizaje relativamente equilibradas en cuanto a los objetivos del aprendizaje, centradas parcialmente en el profesor y en la ejecución de tareas planteadas, exclusivamente, en situaciones matemáticas, mediante enfoques diferentes, y, por otro lado, bajo nivel de dominio de los estudiantes.

Según el modelo de OTL-CM elaborado, las oportunidades de aprendizaje proporcionadas a los estudiantes corresponden a las del segundo nivel de la THE, con la excepción de que no se han observado conexiones entre las Matemáticas y el mundo real, que según las relaciones consideradas podrían favorecer a la formación de las competencias matemáticas.

Hemos documentado que los estudiantes han tenido la oportunidad de aprender los conceptos y hechos matemáticos como unidades de información interconectados e 
interrelacionados entre sí, que tienen sentido solamente dentro del cuerpo matemático; de aprender a manejar y comunicar el lenguaje formal y simbólico, y las operaciones; de elaborar y seguir encadenamientos de axiomas y teoremas; de resolver problemas no rutinarios y no triviales. Esta manera de presentar las Matemáticas está totalmente desconectada y alejada de la experiencia y de la realidad del estudiante que, a pesar de que le permite actuar con cierta seguridad en el campo puramente matemático, le hace sentir impotente y confuso ante los problemas contextualizados y no familiares.

En general, se puede ver en las estrategias didácticas de Mery dos patrones de actuación. El primero, cuando tiene protagonismo principal en cuanto a la exposición de los tópicos, procedimientos y soluciones mediante la estrategia explicación o entrenamiento; el segundo, cuando hace participar a los estudiantes, planteándoles preguntas, invitando a trabajar en tareas, y les da la oportunidad de explorar y llegar a alguna idea o solución, compartir sus pensamientos, empleando las estrategias didácticas cuestionamiento, exploración y participación.

El papel del estudiante, a su vez, también es doble: atiende a las explicaciones de la profesora o contesta, explora, resuelve. Por tradición, los propios estudiantes leen el enunciado de la tarea, organizan los datos en la pizarra, realizan el dibujo correspondiente, con la ayuda de la profesora cuando es preciso. Se les da la oportunidad de plantear el problema, pensar y elegir entra las posibles estrategias de resolución, pronunciar sus ideas al respecto y proceder de modo autónomo, aunque siempre sancionado por la profesora. No obstante, a pesar de que se les da la oportunidad de realizar ciertas actividades, ese papel activo no es exactamente el que, según Mialaret (1984), permite al estudiante mismo, a partir de su experiencia real, descubrir el edificio matemático, siendo la profesora quien conduce este descubrimiento, lo que conlleva el peligro de caer en una forma de enseñanza dogmática.

Como apreciamos en el análisis de las sesiones, Mery ha proporcionado a los estudiantes la oportunidad de trabajar, mayormente, en tareas del grupo de conexión, pero también en las del de reproducción y reflexión. Por tanto, los estudiantes han tenido la oportunidad de resolver problemas con demanda cognitiva diversa.

Por otra parte, todas las tareas son planteadas, exclusivamente, en situaciones matemáticas (formales) y no sólo las que se relacionan con los tópicos grabados u observados, sino las tareas que manejan durante todo el curso. Por tanto, no se ha dado la oportunidad de desarrollar las habilidades de matematización, precisamente las que necesitaban los estudiantes para abordar problemas realistas de la prueba. 
No es de extrañar que los estudiantes hayan tenido dificultades para entender los enunciados de los problemas realistas, ya que las tareas presentadas en los libros de texto tienen todos los datos necesarios presentes, rara vez se acompañan con alguna representación gráfica y, además, no hace falta pensar en el enunciado sino en la solución.

Acorde con las tradiciones en la enseñanza de matemáticas en Armenia (su descripción se puede consultar en Zakaryan (2011)), en lo sucedido en las clases de matemáticas es fácil reconocer las prioridades y pretensiones que tienen en la formación matemática de los estudiantes y su divergencia con las prioridades del proyecto OCDE/PISA. Es obvio que las oportunidades de aprendizaje proporcionadas en el Caso 2 no hayan favorecido el desarrollo de las competencias matemáticas, evaluadas en este proyecto ${ }^{5}$. De este modo, los resultados relativos al Caso 2 sugieren que las OTL proporcionadas a los estudiantes les han permitido dominar y manejar los conocimientos y destrezas matemáticos curriculares, sin embargo, han limitado la capacidad de aplicarlos fuera del contexto escolar.

\section{Conclusiones}

Como hemos mencionado, los dos casos estudiados han tenido un doble interés: particular, por una parte, pues nos interesamos en aprender acerca de dos realidades particulares concretas, y general, dado que estos dos casos nos han servido como instrumentos para generar y comprobar el modelo teórico.

En cuanto a los resultados obtenidos en el Caso 1, han mostrado que el proceso de enseñanza-aprendizaje de matemáticas en el cual se proporcionan unas OTL limitadas en la conjunción con otros factores desfavorables, como el conocimiento previo insuficiente de los estudiantes, la desmotivación del profesor y de los estudiantes, está condenado al fracaso.

Por otra parte, los resultados del Caso 2 revelan que, incluso cuando se dan unas OTL relativamente equilibradas, pero sin salir del plano matemático, la capacidad de los estudiantes para aplicar sus conocimientos fuera del contexto escolar sigue siendo insatisfactoria.

La diferencia que observamos, es que en el Caso 1 los estudiantes carecen de los conocimientos y destrezas curriculares y, consecuentemente, no son capaces de aplicarlos;

\footnotetext{
${ }^{5}$ Así, no se ha enseñado a reconocer regularidades en su vida cotidiana, a elegir de los numerosos datos los más importantes, a expresar, defender o criticar su punto de vista utilizando inteligentemente los argumentos matemáticos, a extraer información de los gráficos, tablas o diagramas, a aplicar sus conocimientos a situaciones del mundo real. Además, los tópicos de la sub-área Incertidumbre relacionados con la probabilidad y estadística no han estado incluidos en la Programación de ninguno de los nueve grados seguidos por estos estudiantes. 
mientras en el Caso 2 dominan los conocimientos y destrezas curriculares, sin embargo, no son capaces de aplicarlos a situaciones de la vida real. Este hecho confirma la premisa de que para una actuación competente es imprescindible un amplio abanico de conocimientos matemáticos y destrezas, sin embargo, esto no es suficiente.

Por otro lado, en el Caso 2 la llamativa ausencia de cualquier conexión de las Matemáticas con el mundo real convence de que aun cuando se movilizan estos conocimientos y destrezas, si quedan exclusivamente en el plano matemático, impiden a los estudiantes transferirlos a las situaciones diferentes de las aprendidas, o sea, abstraer del contenido matemático y generalizar sus conocimientos a situaciones de la vida real.

Cabe destacar la importancia de atender las diferentes facetas del conocimiento matemático a lo largo de la formación escolar ya desde la enseñanza primaria. Particularmente, la inseguridad y la lentitud en el cálculo y en el manejo de los resultados numéricos que notamos en las actuaciones de los estudiantes, son fruto de escasas oportunidades anteriores para una asimilación íntegra de los conocimientos y para el cálculo mental, que han impedido la evolución rápida posterior de los estudiantes (MIALARET, 1984). Asimismo, por ejemplo, la falta de las habilidades para un procedimiento matemático tan básico como despejar, reside en las experiencias poco significativas anteriores de los estudiantes al respecto.

Por otra parte, en el Caso 1, la ausencia entre las OTL de los focos matemáticos tan importantes como problem solving y reasoning, e hincapié, a su vez, en los conceptos y procedimientos sin debida conexión entre entes matemáticos, ha provocado graves lagunas en los conocimientos y destrezas de los estudiantes. Es notable la diferencia en el uso del lenguaje simbólico, teoremas y axiomas, la seguridad en el dominio de resultados numéricos, procedimientos y técnicas, en el planteamiento y resolución de problemas en el aula de los estudiantes del Caso 2, que han tenido la oportunidad de experimentar, de manera relativamente equilibrada, los diferentes procesos y habilidades matemáticos, aunque, como mencionamos, exclusivamente en el plano matemático.

En cuanto a las estrategias didácticas (explicación, entrenamiento, cuestionamiento, participación y exploración) que condicionan la interacción entre el profesor y el estudiante y determinan el papel de cada uno en el proceso de enseñanza-aprendizaje, en el Caso 1, los estudiantes han tenido pocas oportunidades para desempeñar un papel activo, y en el Caso 2 han tenido más oportunidades, sin embargo, su papel no ha sido suficientemente activo, ya que se combinaba con la intervención autoritaria y sancionadora de la profesora. Este hecho sugiere que las escasas oportunidades para una participación activa de los estudiantes en la 
construcción de su propio conocimiento no han sido suficientes para favorecer la actuación competente de los estudiantes.

Por otra parte, se ha confirmado una fuerte relación de las CM de los estudiantes con la oportunidad de resolver cierto tipo de tareas. En el Caso 1 observamos la ejercitación, sobre todo, de tareas del grupo de reproducción, planteadas, mayormente, en situaciones matemáticas; en el Caso 2, aunque prioritariamente resuelven tareas del grupo de conexión, sin embargo, tan solo en situaciones matemáticas. De ahí, es evidente la importancia tanto de la demanda cognitiva de la tarea como su presentación en diferentes situaciones y contextos.

El propósito principal en la formación matemática debe ser conseguir una sincronía entre lo formal y lo cotidiano, o de acuerdo con Schoenfeld (1988), facilitar a los estudiantes a aprender a pensar matemáticamente, lo que implica dominar tanto procedimientos y hechos, como comprender las conexiones entre ellos y ser capaz de aplicar con flexibilidad y significado el conocimiento matemático formal en varias situaciones.

De este modo, los resultados relativos al Caso 1 confirman que las escasas OTL que favorecen el aprendizaje significativo de las matemáticas limitan tanto la adquisición de los conocimientos y destrezas matemáticos básicos como la capacidad de aplicarlos en situaciones de la vida real, estando lejos así del principal propósito de la formación matemática, en este caso no se ha cumplido ni siquiera la condición necesaria. En lo que se refiere al Caso 2, estamos ante un hecho casi universal que "constituye un dramático fracaso de la instrucción" (SCHOENFELD, 1988, p. 7) cuando los estudiantes capaces de realizar operaciones simbólicas en un contexto del aula, demostrando dominio de una materia determinada, no logran conectar los procedimientos formales con los objetos del mundo real. En este Caso, aunque se cumpla la condición necesaria para aprender a pensar matemáticamente, en términos de este autor, la condición suficiente de ser capaz de aplicar con flexibilidad y significado el conocimiento matemático formal en una variedad de situaciones, deja bastante que desear para ser cumplida.

Ahora bien, como hemos visto, el objetivo de aprendizaje, en los dos casos estudiados, no ha sido que los estudiantes adquirieran tales competencias, por lo que, no es de extrañar que las actividades de ambos profesores no fuesen dirigidas especialmente para su desarrollo y parece evidenciarse que, en el caso contrario, las mismas actividades de los profesores no hubieran sido adecuadas.

En síntesis, estimamos que nuestro estudio aporta concreción a las posibles consecuencias que determinadas acciones de un profesor tiene en el aprendizaje de sus estudiantes. 


\section{Referencias}

ANDREWS, P.; CARRILLO, J.; CLIMENT, N. Proyecto "METE”: El foco matemático. In: SIMPOSIO DE LA SOCIEDAD ESPAÑOLA DE INVESTIGACIÓN EN EDUCACIÓN MATEMÁTICA, $9^{\text {th }}, 2005$, Córdoba. Proceedings... Córdoba: Universidad de Córdoba, 2005, p. 131137. CD-ROM

BARDIN, L. El análisis de contenido. Madrid: Akal Ediciones, 1996.

BERICAT, E. La integración de los métodos cuantitativos y cualitativos en la investigación social. Barcelona: Ariel, 1998.

CARRILLO, J.; CONTRERAS, L.C.; ZAKARYAN, D. Avance de un modelo de relaciones entre las oportunidades de aprendizaje y competencias matemáticas. Bolema, Rio Claro (SP), v. 27, n. 47, p. 779-804, Dec. 2013.

COHEN, L; MANION, L. Métodos de investigación educativa. Madrid: La Muralla, 2002.

HIEBERT, J.; GROUWS, D. The effects of classroom mathematics teaching on students' learning. In: LESTER, F. (Ed.). Handbook of Research on Mathematics Teaching and Learning. NCTM: Information Age Publishing, 2007. p. 371-404.

INSTITUTO NACIONAL DE EVALUACIÓN Y CALIDAD DEL SISTEMA EDUCATIVO (INECSE). PISA 2003. Pruebas de Matemáticas y de Solución de Problemas. Madrid: MEC. 2005.

LINACRE, J. A User's Guide to Winstep. Rasch-Model Computer Programs. 2006. Disponible en: www.winsteps.com. Acceso en: 17 sept. 2009.

MCDONNELL, L. Opportunity to learn as a research concept and a policy instrument. Educational Evaluation and Policy Analysis, Washington, v. 17, n. 3, p. 305-322, Sept. 1995.

MIALARET, G. Las Matemáticas: cómo se aprenden, cómo se enseñan. Madrid: Visor, 1984.

ORGANIZACION PARA COOPERACION Y DESARROLLO ECONOMICO (OCDE). The PISA 2003 Assessment Framework - Mathematics, Reading, Science and Problem Solving Knowledge and Skills. Paris: OECD, 2003.

ORGANIZACION PARA COOPERACION Y DESARROLLO ECONOMICO (OCDE). Marcos teóricos de PISA 2003. Conocimientos y destrezas en Matemáticas, Lectura, Ciencias y Solución de problemas. Madrid: MEC, 2004.

PIAGET, J. To understand is to invent: The future of education. Nueva York: Viking, 1973.

PRIETO, G.; DÍAS, A. Uso del modelo de Rasch para poner en la misma escala las puntuaciones de distintos tests. Actualidades en Psicología, Costa Rica, v. 19, n. 106, p. 5-23, Dec. 2003.

SCHOENFELD, A. When good teaching leads to bad results: the disasters of "well-taught" mathematics courses. Educational Psychologist, UK: Routledge, v. 23, n. 2, p. 145-166. Aug. 1988.

SCHOENFELD, A. On modeling teaching. Issues in Education, Ipswich, MA, USA, v. 4, n. 1, p. 149-162, Jan. 1998.

SIMON, M. Reconstructing mathematics pedagogy from a constructivist perspective. Journal for Research in Mathematics Education, Reston, v. 26, n. 2, p. 114-145, Jan. 1995. 
STAKE, R. Investigación con estudio de casos. Madrid: Morata, 2007.

STEVENS, F.; GRYMES, J. Opportunity to learn: Issues of equity for poor and minority students. Washington, DC: National Center for Education Statistics, 1993.

ZAKARYAN, D. Oportunidades de aprendizaje y competencias matemáticas de estudiantes de 15 años. Un estudio de casos. 2011. 446 f. Tesis (Doctorado en Didáctica de la Matemática) Facultad de Ciencias de la Educación, Universidad de Huelva, Huelva, 2011.

Submetido em Março de 2013. Aprovado em Junho de 2013. 